\title{
PERFIL DE PESSOAS COM DEFICIÊNCIAS FÍSICO-MOTORAS CONCORRENTES PELA AÇÃO AFIRMATIVA B DE INGRESSO AO ENSINO SUPERIOR
}

\author{
Aline Sarturi Ponte, Francini Jacques de Souza, Jodeli Pommerehn, Miriam Cabrera Corvelo Delboni
}

\section{RESUMO}

Resumo: 0 estudo buscou analisar o perfil dos candidatos com deficiência físico-motora concorrentes da Ação Afirmativa B no Concurso Vestibular 2012 de uma Instituição Ensino Superior (IES) pública do interior do Rio Grande do Sul. A importância deste está na exploração das características biopsicossociais que definem sujeitos aptos à reserva de vagas, bem como em conhecer as barreiras ambientais. Este estudo é documental, descritivo, retrospectivo e quantitativo. Foram analisados 41 Check List resumido da Classificação Internacional de Funcionalidade, Incapacidade e Saúde (CIF), dos quais 22 foram considerados aptos $\mathrm{e} 11$ inaptos à reserva de vagas. Ambos os grupos apresentaram deficiências nas Funções e Estruturas do Corpo relacionadas principalmente ao Movimento, o que os difere são as limitações nas Atividades e Participação e nas barreiras Ambientais. A CIF permitiu compreender o processo de funcionalidade e incapacidade humana a partir da relação da participação social do candidato, bem como estar de acordo com as leis que regem o acesso por meio da reserva de vagas para pessoas com necessidades especiais.

Descritores: Pessoas com Deficiência; Instituição de Ensino Superior; Classificação Internacional de Funcionalidade; Incapacidade e Saúde.

Profile of people with physical and motor disabilities competitors for Affirmative Action B ticket to higher education

Abstract: The study sought to analyze the profile of the candidates with physically and motor deficiency competing in Affirmative Action B in the 2012 Vestibular Contest of a Public Higher Education Institution in the interior of Rio Grande do Sul. The importance of this is in exploring the biopsychosocial characteristics that define subject to the reserve of vacancies, as well as to know the environmental barriers. This study is documentary, descriptive, retrospective and quantitative. A total of 41 summary check lists of the International Classification of Functioning, Disability and Health (ICF) were analyzed, of which 22 were considered fit and 11 were unfit for vacancy reservations. Both groups had deficiencies in the Functions and Body Structures related mainly to the Movement, what differentiated them are the Limitations in Activities and Participation and Environmental Barriers. The ICF made it possible to understand the process of human functionality and incapacity based on the relation of the social participation of the candidate, as well as being in accordance with the laws that govern access by reserving places for people with special needs.

Descriptors: Disabled people; Higher Education Institutions; International Classification of Functioning; Disability and Health.
Francini Jacques de Souza, Terapia Ocupacional Formada(o) em Terapia Ocupacional pela (o) UFSM que fica na cidade de Santa Maria, estado Rio Grande do Sul, País Brasil.

Aline Sarturi Ponte, Mestre em Distúrbios da Comunicação Humana pela Universidade Federal de Santa Maria UFSM Formada(o) em Terapia Ocupacional pela(o) UNIFRA que fica na cidade de Santa Maria, estado Rio Grande do Sul, País Brasil.

Jodeli Pommerehn, Mestranda em Distúrbios da Comunicação Humana pela Universidade Federal de Santa Maria UFSM Formada(o) em Terapia Ocupacional pela(o) UFSM que fica na cidade de Santa Maria, estado Rio Grande do Sul, País Brasil. Miriam Cabrera Corvelo Delboni, Doutoranda em em Desenvolvimento Regional, Universidade de Santa Cruz do Sul - Unisc Formada(o) em Terapia Ocupacional pela(o) Puccamp que fica na cidade de Campinas, estado São Paulo, País Brasil. 
As pessoas com deficiência enfrentam dificuldades para chegar às Instituições de Ensino Superior (IES) brasileiras, pois além de se depararem com obstáculos estruturais, como por exemplo, pouca ou inexistência de acessibilidade, também encontram barreiras atitudinais, seja através do desinteresse ou insuficiência de informações presentes nas estruturas curriculares dos cursos, ou pelo despreparo dos docentes1. No Brasil no ano de 2008, haviam dezenove IES Públicas com algum tipo de Ação Afirmativa para pessoas com deficiência. Dentre as 59 IES Federais brasileiras, apenas sete reservavam vagas para deficientes2.

Cabe ressaltar que estes obstáculos não se restringem somente ao Ensino Superior (ES), estão presentes em todas as etapas da educação3. Sabe-se que esta situação fere um direto básico, que é o acesso à educação assegurado pelo Art. 208, parágrafo III da Constituição Federal (CF) brasileira, o qual defende o acesso à educação regular de ensino4. Com intuito de fortalecer o artigo da CF, o Governo Federal vinculado ao Ministério da Educação implantou em 24 de outubro de 1989, a Lei $n^{0} 7.853$. Este documento em seu Art. $2^{\circ}$ parágrafo I que defende 0 acesso da pessoa com deficiência a educação, e no parágrafo IV institui a formação e a qualificação de recursos humanos (professores, técnicos e outros profissionais) para atender às necessidades desta população5.

A Lei de Diretrizes e Bases da Educação Nacional (LDB), Lei no. 9.394 de 20 de dezembro de 1996, a qual reservou um capítulo exclusivamente para o tratamento da Educação Especial. Em seu Art. $n^{\circ} 59$, preconiza que os sistemas de ensino devem assegurar aos alunos; currículo, métodos, recursos e organização específicos para atender às suas necessidades6. Ou seja, deve abranger uma modalidade de educação, da mesma forma voltada para a formação geral do sujeito, visando ao desenvolvimento social da pessoa com deficiência.

O Decreto n 3.298 de 20 de dezembro de 1999 em seu Art. 27 institui que as IES "deverão oferecer adaptações de provas e os apoios necessários, previamente solicitados pelo aluno [...]" com deficiência7. 0 inciso ${ }^{10}$ deste mesmo decreto abrange o sistema geral do processo seletivo para ingresso em cursos superiores e no inciso $2^{\circ}$ institui que o Ministério da Educação "no âmbito da sua competência, expedirá instruções para que os programas de educação superior incluam nos seus currículos conteúdos, itens ou disciplinas relacionados à pessoa [...]" com deficiência7.

Também o Decreto 6.949 de 25 de agosto de 2009 Art. nº 24, parágrafo 5, garante as pessoas com deficiência a igualdade de condições no acesso ao ensino superior, treinamentos profissionalizantes, educação continuada, respeitando a vocação destes sujeitos. Este ainda, coloca como dever dos Estados as adaptações necessárias para o acesso8.

Para assegurar os direitos desta população em de 06 de julho de 2015 é instituída a Lei 13.146 - Estatuto da Pessoa com Deficiência, que eu seu Art. $8^{0}$ estabelece que, é de dever do estado, da sociedade e da família garantir o acesso à educação, assim como a outros direitos defendidos pela CF Brasileira, Convenção sobre os Direitos das Pessoas com Deficiência e outros documentos legais que garantam o bem-estar pessoal, social e econômico desta população9.

No Capítulo IV (Do Direito a Educação), Art. nº 28, inciso XIII garante o "acesso à educação superior e à educação profissional e tecnológica em igualdade de oportunidades e condições com as demais pessoas"9:34. E o Art. 30 complementa com as medidas que devem ser adotadas pelas IES para acesso ao processo seletivo e permanência da pessoa com deficiência no curso, são exemplos de medidas: 0 atendimento prioritário nas dependências da IES, a oportunidade de o candidato especificar os recursos de acessibilidade e de tecnologia assistiva necessários para sua participação no processo seletivo, dilatação do tempo considerando as necessidades dos sujeitos, etc9. Atualmente no Brasil a Lei de Cotas, Lei no 12.711, regulamentada pelo Governo Federal em 29 de agosto de 2012, não traz em sua redação a obrigatoriedade de reserva de vagas para pessoas com deficiência10, mas algumas instituições superiores estão adotando a reserva de vagas a pessoas com deficiência em decorrência do Decreto $n^{0} 3.298 / 99$

Com base no exposto, este estudo tem como objetivo analisar o perfil dos candidatos com deficiência físicomotora concorrentes pela Ação Afirmativa B no Concurso Vestibular 2012 de uma IES do interior do Rio Grande do Sul. A importância de tal abordagem está na exploração das características biopsicossociais que definem sujeitos aptos e não aptos à reserva de vagas, bem como as barreiras e dificuldades encontradas por esses sujeitos no cotidiano. Pois com isso pode-se ter uma visão ampliada das pessoas com deficiência que buscam uma vaga no ensino superior contribuindo para a (re) formulação de medidas inclusivas.
Este estudo é documental, descritivo, retrospectivo e quantitativo. A coleta de dados ocorreu no período fevereiro a setembro de 2013, na qual foram analisados questionários de 41 pessoas com idade superior a 18 anos, com deficiência físico-motora, concorrentes pela Ação Afirmativa B no Concurso Vestibular do ano de 2012 de uma IES pública do interio do Rio Grande do Sul. A coleta de dados iniciou após a autorização do Comitê de Ética em Pesquisa em Seres Humanos, da Universidade onde se realizou o estudo, sob parecer número 222.242

A IES envolvida neste estudo localiza-se na região central do Rio Grande do Sul, e conta com o Programa de Ações Afirmativas implantado em 3 de agosto de 2007 a partir da aprovação da Resolução 011/07, que trata de um conjunto de medidas para a promoção de inclusão social e democratização de acesso ao ensino superior, envolvendo 0 ingresso e reingresso da universidade, esta resolução em seu Art. $n^{0} 4$ da Resolução N.011/07 reserva uma porcentagem de $5 \%$ das vagas em cada curso de graduação para Ação Afirmativa B, sendo estas vagas destinadas as pessoas com deficiência11.

Com a aprovação deste programa, a partir do Concurso de Vestibular do ano de 2008, a universidade passou a destinar um número específico de vagas para: 1) afro-brasileiros; 2) alunos que cursaram todo o ensino fundamental médio em escolas públicas; 3 ) pessoas com deficiência; e 4) indígenas, tendo em vista a necessidade de democratizar 0 acesso ao Ensino Superior público no país11.

A partir de então, esta instituição estruturou um programa permanente de acompanhamento e de apoio sociopedagógico aos estudantes cotistas, coordenado por comissão constituída especificamente para esse fim. Criou também o Observatório de Ações Afirmativas, que está subordinado à Pró-Reitoria de Graduação (PROGRAD) e é destinado a observar o funcionamento das Ações Afirmativas, avaliar seus resultados, identificar aspectos que prejudiquem sua eficiência e sugerir ajustes e modificações, apresentando ao Conselho Universitário, relatórios anuais de avaliação. Esta Comissão de Acompanhamento do Programa é composta por 22 pessoas, entre professores, representantes da PROGRAD, Seção Sindical dos Docentes da instituição, Associação dos Servidores da IES, Comissão Permanente de Vestibular (COPERVES), Núcleo de Estudos Afro-Brasileiros, Diretório Central de Estudantes (DCE), além de representantes da Comunidade Externa11.

A fim de que o processo eletivo promova a inclusão de forma justa e ética, ao longo deste período de inserção da Resolução 011/2007, o acesso e permanência na instituição vêm tomando forma para que a inclusão no ensino superior não se torne excludente. Desde sua implantação o ingresso de pessoas com deficiência vem sendo reformulado. Nos anos anteriores a 2012, o candidato apresentava 0 atestado médico com o código da Classificação Internacional de Doenças e Problemas Relacionados à Saúde (CID), associado aos demais documentos após a realização do processo de seleção, o que ocasionava por vezes o constrangimento dos candidatos que haviam se classificado inadequadamente pela Ação Afirmativa B12.

Então, a partir de 2012 ocorreram reformulações no processo, assim no concurso do ano de 2013 os candidatos passaram por entrevistas previamente agendadas, a partir desta os concorrentes foram classificados como aptos a seguirem no processo pela Ação Afirmativa B e os considerados inaptos passaram para outra Ação afirmativa, escolhida como segunda opção no momento da inscrição ou concorriam pelo Sistema Universal.

Para a entrevista dos candidatos com deficiências foram divididos em dois grupos: 1) candidatos com deficiências físico-motora (Mielomeningocele, Paralisia Cerebral e amputações); e 2) candidatos com deficiências sensoriais (visuais e auditivas)

A entrevista dos candidatos com deficiência físico-motora foi embasada pelo Check List resumido da Classificação Internacional de Funcionalidade, Incapacidade e Saúde (CIF). Este instrumento foi utilizado pela primeira vez na instituição e aplicado pela Comissão que é composta por um docente do Curso de Terapia Ocupacional, um docente do Curso de Fisioterapia, dois docentes do Curso de Educação Especial e um representante da COPERVES.

Para as pessoas com deficiências sensoriais foi estabelecida uma Comissão de Verificação que analisaria os documentos do candidato e 0 atestado médico, este deveria ser emitido nos últimos doze meses, assinado por um médico especialista na área da deficiência alegada pelo candidato, contendo o grau ou nível de deficiência, o código correspondente ao CID, bem como a provável causa dessa deficiência. Deveria ainda conter nome legível e o número do Conselho Regional de Medicina (CRM) do médico que forneceu o atestado13.

Para coleta de dados deste estudo realizou-se a análise documental: i) da lista de inscritos disponibilizada pela 
COPERVES e ii) do CheckList da CIF respondidos pelas pessoas com deficiência físico-motora. Na primeira coletou-se dados como sexo, idade, tipo de deficiência e opção de curso. Na segunda, coletou-se informações das duas partes do Check List da CIF: a Parte 1 - Funções e Estruturas do Corpo - Atividade e Participação (Funcionalidade e Incapacidade) e Parte 2 - Fatores Contextuais (Fatores Ambientais - Pessoais)14.

"A funcionalidade e a incapacidade associados aos estados de saúde são classificados na CIF"14:7. Esta classificação abrange os fatores físicos e sociais, considerando as diferentes percepcões culturais e atitudes em relação à deficiência, a disponibilidade de serviços e a legislação15,16. Sendo assim, esta classificação "proporciona uma descrição de situações relacionadas às funções humanas e às suas restrições"17:5. 0 uso da CIF não se restringe à área da saúde, outras áreas podem se beneficiar, como, por exemplo, educação, a assistência social, advocacia, previdência social14,16

Para expor os aspectos relacionados a Funcionalidade e Incapacidade a CIF conta com uma relação de qualificadores, é atribuído por meio de uma escala genérica de 0 (zero) a 4 (quatro), onde o Zero corresponde a nenhuma deficiência, 1 (um) - deficiência leve, 2 (dois) - deficiência moderada, 3 (três) - deficiência grave, 4 (quatro) - deficiência completa. Há, ainda, os qualificadores 8 (oito) e 9 (nove), utilizados, para problema - não especificado - para informações insuficientes; e - não aplicável - para quando o código for inapropriado para o sujeito envolvido. Para os Fatores Ambientais e Pessoais utilizam-se os qualificadores de barreiras ou facilitadores, onde (zero - nenhum (a) facilitado barreira; -1 (barreira leve), -2 (barreira moderada), -3 (barreira considerável) e -4 (barreira completa), respectivamente; +1 (facilitador leve), +2 (facilitador moderado), +3 (facilitador considerável) e +4 (facilitador completo), respectivamente16,18

Os dados coletados foram todos tabulados no Software Excel 2010 e para o tratamento realizou-se uma análise descritiva, utilizando o Software Statistica 9.1 .

\section{Resultados}

A partir da análise das listas dos candidatos inscritos na Ação Afirmativa B com deficiência físico-motora observouse que dos 41 candidatos, $68 \%$ são do sexo masculino. A média de idade apresentada pelos inscritos foi de 23,3 anos, sendo a idade mínima 18 anos e a máxima 48 anos.

Quanto à escolha do curso, observa-se que 0 curso de Medicina foi o mais concorrido entre os candidatos com deficiência físico-motora concorrentes à Ação Afirmativa B. Foram 17 candidatos ao curso de Medicina, sendo que destes oito $(45 \%)$ foram considerados aptos pela Comissão, seis $(33 \%)$ inaptos e três $(22 \%)$ não compareceram à entrevista. Outros cursos como Direito Diurno tiveram três candidatos, Direito Noturno quatro e Administração Noturno dois, obtiveram a procura superior a uma pessoa (FIGURA 1).

FIGURA 1 - Cursos solicitados pelos candidatos inscritos à Ação Afirmativa B com deficiência físico-motora n=41

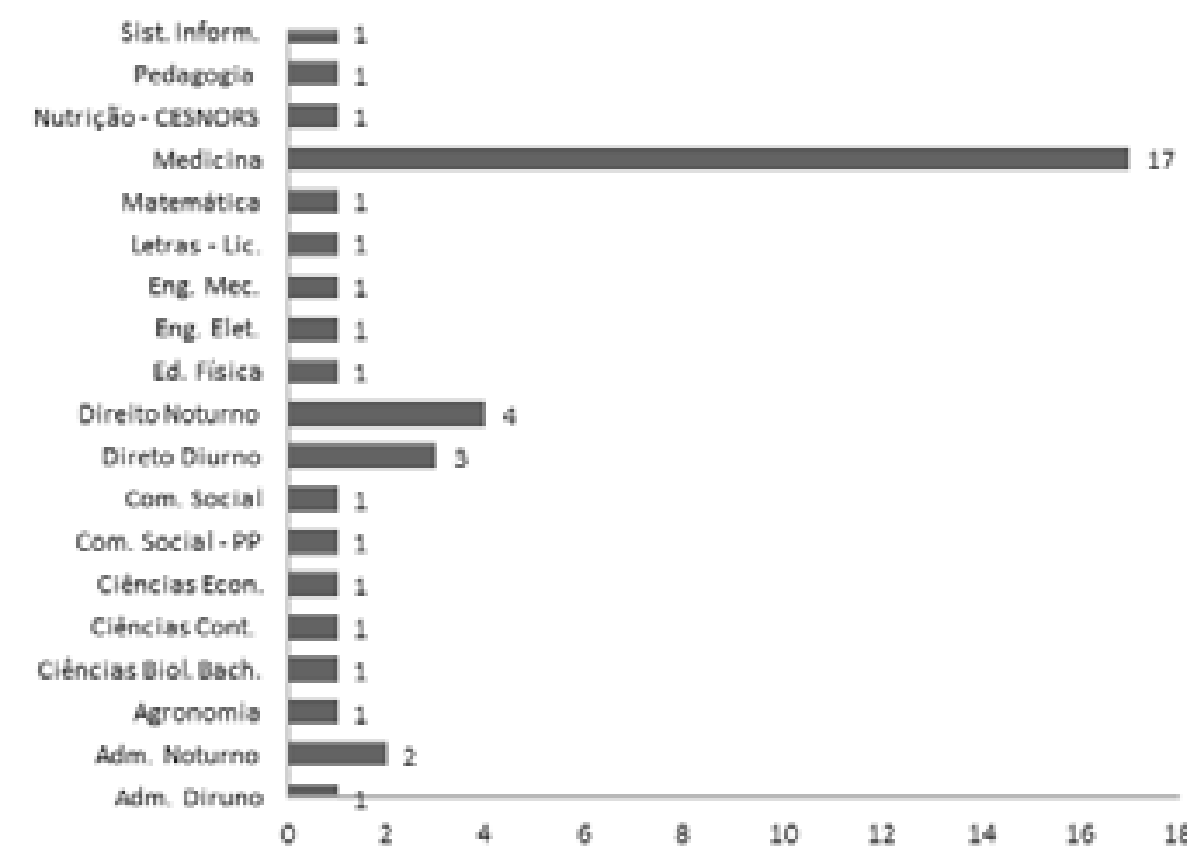

Legendas: Adm. -Administração
Bach. - Bacharelado Biol., - Biológicas, Cont. - Contábeis, CESNORS - Centro de Educação Superior Norte do Rio Grande do Sul, Com. - Comunicação, Econ. - Econômicas, Ed. - Educação, Elet. - Elétrica, Eng. - Engenharia, Inform. - Informação, Lic. - Licenciatura, Mec. - Mecânica, Not. - Noturno, Sist. - Sistema, PP - Publicidade e Propaganda.

Fonte: elaborados pelas autoras.

Dos 41 candidatos com deficiência físico-motoras, $22(54 \%)$ foram considerados aptos, $11(27 \%)$ inaptos e oito (19\%) pessoas não compareceram à entrevista.

No que se refere às Funções Corporais, observou-se que tanto os sujeitos aptos (56\%), quanto os inaptos (45\%) referem limitações nas Funções Neuromusculoesqueléticas e relacionadas ao Movimento (TABELA 1).

TABELA 1 - Deficiências das Funções Corporais dos candidatos aptos e inaptos concorrentes a vagas da Ação Afirmativa $B, n=33$.

\section{Componentes}

Funções Sensoriais e Dor

Funções da Voz e da Fala

Funções dos Sistemas Cardiovascular, Hematológico,

Imunológico e Respiratório

Funções dos Sistemas Digestivo, Metabólico e Endócrino

Funções Geniturinárias e Reprodutivas

Funcões Neuromusculoesqueleticas e relacionadas ao Mor

Funções da Pele e Estruturas Relacionadas

Outras Funções

Não apresentaram o nível completo de deficiência

Não Apresentaram nas Funções do Corpo

$\begin{array}{cc}\text { Sujeitos Aptos } & \text { Sujeitos Inaptos } \\ 2(6 \%) & - \\ 2(6 \%) & - \\ 1(3 \%) & - \\ 3(9 \%) & - \\ 4(11 \%) & - \\ 19(56 \%) & 4(45 \%) \\ 1(3 \%) & - \\ - & 1(11 \%) \\ - & 3(33 \%) \\ 2(6 \%) & 1(11 \%)\end{array}$

Com relação às deficiências das Estruturas Corporais, observou-se que ambos os grupos apresentam limitações associadas às Estruturas Relacionadas ao Movimento, sendo mencionado por 14 (43\%) sujeitos aptos e seis (60\%) dos sujeitos inaptos, como apresentado na Tabela 2.

TABELA 2 - Deficiência apresentada pelos candidatos nas Estruturas Corporais mais comprometidas dos candidatos $n=33$

\section{Componentes}

Estruturas Relacionadas ao Movimento

Estruturas do Sistema Nervoso

Estruturas Relacionadas ao Sistema Geniturinário e Reprodutivo

Estruturas de Pele e Relacionadas

Estruturas Relacionadas a Voz e Fala

Não apresentaram nenhuma deficiência na estrutura

Outras Quaisquer

Não apresentaram deficiências em nível completo

Fonte: elaborados pelas autoras.

$\begin{array}{cc}\text { Sujeitos Aptos } & \text { Sujeitos Inaptos } \\ 14(43 \%) & 6(60 \%) \\ 8(24 \%) & 1(10 \%) \\ 3(9 \%) & - \\ 1(3 \%) & - \\ 1(3 \%) & - \\ 3(9 \%) & - \\ - & 2(20 \%) \\ 3(9 \%) & 1(10 \%)\end{array}$

Quanto ao item da CIF que aborda as Atividades e as Restrições à Participação, pode-se verificar que os candidatos aptos apresentaram maiores limitaçoes nos atos que envolvem a Mobilidade (59\%) quando comparados com os inaptos (TABELA 3). 

$n=33$.

TABELA 3 - Limitações encontradas pelos candidatos nas Atividades e Restrição à Participação dos candidatos

\section{Componentes}

Mobilidade

Tarefas e Demandas Gerais

Cuidado Pessoal

Comunicação

Não apresentam nível completo de deficiência

Não apresentaram nenhuma restrição

Fonte: elaborados pelas autoras.

Os Fatores Ambientais apresentados como barreiras pelos candidatos aptos foram os Produtos e Tecnologia usados em projeto, arquitetura e construção de edifícios para uso público, referido por $64 \%$ dos candidatos, $36 \%$ dos candidatos não relataram barreiras. Dos candidatos inaptos, $82 \%$ não encontram nenhuma barreira e $18 \%$ mencionaram que encontram barreiras nos Produtos e Tecnologia usados em projeto, arquitetura e construção de edifícios para uso público.

\section{Discussão}

O estudo revelou que dos 41 candidatos à Ação Afirmativa B na seleção do Concurso de Vestibular do ano de 2012 somente 33 compareceram às entrevistas. Destes a maioria é do sexo masculino, a média de idade dos participantes foi de 23,3 anos e curso mais escolhido foi o de Medicina (Figura 1). A faixa etária dos candidatos é compatível com a média de idade dos jovens que ingressam no ensino superior, assim indicando que os com deficiência também estão conseguindo ingressar. O Censo realizado pelo Instituto Brasileiro de Geografia e Estatística - IBGE no ano de 2010 destacou que 45,6 milhões de pessoas declararam possuir alguma deficiência, o que corresponde a $23,9 \%$ da população brasileira, deste total apenas $6,7 \%$ destas pessoas, com mais de 15 anos ingressou em um curso superior 19 , o que corresponde a um número muito pequeno comparado com a população total de pessoas com deficiência.

Cabe ressaltar que dos 33 candidatos com deficiência físico-motora somente 22 foram considerados aptos e 11 inaptos à reserva de vagas. Observou-se que as Funções Corporais (Tabela 1) mais afetadas foram as relacionadas aos Sistema Neuromusculoesqueléticos e ao Movimento, tanto dos candidatos aptos como dos inaptos. Bem como, as Estruturas do Corpo (Tabela 2) relacionadas principalmente ao Movimento e Sistema Nervoso. Esse resultado já era esperado já que foram selecionados candidatos com deficiência físico-motora, porém podemos apontar com esse dado a transição de uma perspectiva individual e médica para uma perspectiva estrutural e social, na qual ter deficiência não se deve somente a anormalidade anatômica, mas sim o quanto a interação com o meio que vive é excludente20.

Como referido anteriormente, ao analisar as limitações das Atividades e Restrições à Participação encontradas pelos candidatos, observou-se que as deficiências físico-motoras dos candidatos aptos acometem em sua maioria a mobilidade, tarefas e demandas gerais e cuidado pessoal (Tabela 3), diferentemente dos candidatos considerados inaptos, os quais enfrentam limitações funcionais mínimas no cotidiano.

Destaca-se que avaliar a seleção das pessoas com deficiência para concorrer a uma vaga especial na universidade pelo Check List da CIF foi fundamental a identificação das limitações à Participação, as barreiras encontradas nos Contextos Ambientais, de forma a contribuir com a percepção diferenciada do censo comum a respeito das características físicas da deficiência.

O termo deficiência ainda gera muitas discussões e opiniões divergentes, assim podemos considerar a definição mais abrangente: "desvantagem ou restrição de atividade provocada pela organização social contemporânea, que pouco ou nada considera as pessoas que possuem lesões e as exclui das principais atividades da vida social"21:8.

Dessa forma devemos ressaltar que os Fatores Ambientais apresentados como barreiras pelos candidatos aptos foram os Produtos e Tecnologia usados em projeto, arquitetura e construção de edifícios para uso público e a maioria dos candidatos inaptos referiram não encontrar nenhuma barreira. Isso novamente reforça a definição de deficiência mencionada acima e sua relação com a participação e as barreiras físicas ou atitudinais que as pessoas com deficiência podem ou não enfrentar em seu cotidiano. Também expõe/indica quais os desafios que as IES terão e como podem oferecer suporte para receber e manter esses alunos. Deve-se pensar como tornar a estrutura física de edifícios, material escolar, ensino acessivel a essas pessoas, mas também na preparação dos movimentos de conscientização e sensibilização para as atitudes pessoais de docentes, acadêmicos e administrativo no acolhimento das necessidades individuais de cada pessoa

Apesar das Leis brasileiras, conforme introdução deste artigo, enfatizarem o acesso e permanência de pessoas com deficiência no ES ainda ocorre um distanciamento entre o que é preconizado e a realidade nas universidades principalmente na acessibilidade, conforme demostrado nesta pesquisa.

Sabe-se que o pensamento de uma sociedade inclusiva está sendo cativada, porém o processo de inclusão de pessoas com deficiência na escola básica ou na universidade não se efetua apenas por decretos ou mesmo leis. Tal processo requer uma mudança profunda na sociedade para que intervenções e medidas sejam eficientes e suficientes para transpor as barreiras que impedem ou restringem o acesso e permanência de pessoas com deficiência na educação22.

Quando alcançarmos a inclusão na educação ela resultará na tão desejável inclusão social22, pois a base da inclusão consiste no conceito de que toda pessoa tem o direito básico à educação e que esta deve levar em conta seus interesses, habilidades e necessidades de aprendizagem.

A inclusão social deve ser vista como fator fundamental para equidade e desenvolvimento da sociedade brasileira que exige que os educadores estejam, constantemente, informados sobre os processos educacionais, necessidades especiais e das possibilidades da tecnologia (assistivas) que proporcionem à pessoa com deficiência maior independência, qualidade de vida e inclusão social.

Conclusão

De um modo geral, os resultados confirmam a importância do uso Check List da CIF durante as entrevistas das vagas de Ação Afirmativa B, pois sem dúvidas o perfil traçado nas categorias, vai de acordo com os grupos estudados, ficando claro que 0 fato de possuir características clínicas de uma deficiência, não necessariamente torna o candidato apto as vagas destinadas a Ação Afirmativa B. É importante a análise do histórico de vida e de seu cotidiano em que as questões de funcionalidade resultam de uma continua interação entre a pessoas e 0 ambiente que a rodeia, facilitando ou impedindo a sua participação social.

O objetivo do estudo não foi comprovar a eficácia do método de avaliação, Checklist da CIF, porém cabe ressaltar que os resultados enfatizam isso ao evidenciar, através da visão ampliada (biopsicossocial) que somente as características clínicas de uma deficiência não são suficientes para o candidato ser apto as vagas destinadas a Ação Afirmativa B. Principalmente que durante a pesquisa não foi encontrado, na literatura, estudo semelhante que tenha utilizado o Checklist, como método avaliativo para candidatos concorrentes a cotas afirmativas.

Além disso, através da CIF, a IES pode identificar questões de acessibilidade e conceituais de deficiência, a fim de que os candidatos que obtiverem o ingresso tenham a condição de permanência no curso escolhido e de conclusão do mesmo. Pois ao entenderem-se as reais necessidades desta população, as IES juntamente com os departamentos coordenações de cada curso, poderão encontrar meios de minimizar as barreiras (físico-arquitetônicas, atitudinais) encontradas pelas pessoas com deficiência no acesso ao ES.

Sugere-se que outros estudos sejam realizados com essa temática a fim de serem produzidas mais evidências acerca do assunto abordado por este estudo.

\section{REFERÊNCIAS BIBLIOGRÁFICAS}

1. Costa VA. Formação de professores e educação inclusiva frente às demandas humanas e sociais Para quê?. In: Miranda TG, Galvão Filho TA. O professor e a educação inclusiva: formação, práticas e lugares. Salvador: EDUFBA; 2012.

2. Ferreira R, Borba A. Mapa das ações afirmativas no ensino superior. Rio de Janeiro: LPP; 2008. 
3. Barbora AS, Duarte ACS. Educação superior para pessoas com deficiência: com a palavra estudantes universitários da cidade de Jequié - BA. Revista FACED, Salvador, 2009; 15: 93-110.

4. Brasil. Constituição da República Federativa do Brasil de 1988. Diário Oficial da República Federativa do Brasil, Brasília, 05 out. 1988. Seção 1.

5. Brasil. Lei n 7.853, de 24 de outubro de 1989. Dispõe sobre o apoio às pessoas portadoras de deficiência, sua integração social, sobre a Coordenadoria Nacional para Integração da Pessoa Portadora de Deficiência - Corde, institui a tutela jurisdicional de interesses coletivos ou difusos dessas pessoas, disciplina a atuação do Ministério Público, define crimes, e dá outras providências. Diário Oficial [da] República Federativa do Brasil, Brasília, DF, 25 out. 1989. Disponível em: <http://www.planalto.gov.br/ccivil_03/leis/L7853.htm> Acesso em: 30 de out. 2015.

6. Brasil. Ministério da Educação. Lei n. ${ }^{\circ}$ 9.394, de 20 de dezembro de 1996. Fixa as Diretrizes e Bases da Educação Nacional. Brasília, DF, 1997.

7. Brasil. Decreto $n^{\circ} 3.298$, de 20 de dezembro de 1999. Regulamenta a lei no 7.853, de 24 de outubro de 1989, que dispõe sobre a política nacional para a integração da pessoa portadora de deficiência, consolida as normas de proteção, e dá outras providências. Diário Oficial [da] República Federativa do Brasil, Brasília DF, 21 dez. 1999. Disponível em: <http://www.planalto.gov.br/ccivil_03/decreto/D3298.htm> Acesso em des. 2015.

8. Brasil. Decreto $n^{\circ}$ 6.949, de 25 de agosto de 2009. Promulga a Convenção Internacional sobre os Direitos das Pessoas com Deficiência e seu Protocolo Facultativo, assinados em Nova York, em 30 de março de 2007. Diário Oficial [da] República Federativa do Brasil, Brasília, DF, 26 ago. 2009, p. 3. Disponível em: <http:// www.planalto.gov.br/ccivil_03/_ato2007-2010/2009/decreto/d6949.htm> Acesso em: 30 de nov. 2012.

9. Brasil. Lei no 13.146, de 6 de julho de 2015. Institui a Lei Brasileira de Inclusão da Pessoa com Deficiência (Estatuto da Pessoa com Deficiência). Disponível em <http://www.planalto.gov.br/ccivil_03/_Ato20152018/2015/Lei/L13146.htm>. Acesso em 17 out. 2015.

10. Brasil. Lei $n^{\circ} 12.711$, de 29 de agosto de 2012. Dispõe sobre o ingresso nas universidades federais e nas instituições federais de ensino técnico de nível médio e dá outras providências. Diário Oficial da União,Brasília, DF, 30 ago. 2012.

11. Universidade Federal de Santa Maria. Resolução n. 011/07, de 3 agosto de 2007. Institui, na Universidade Federal de Santa Maria, o Programa de Ações Afirmativas de Inclusão Racial e Social e revoga a Resolução n. 009/07. Gabinete do Reitor da Universidade Federal de Santa Maria, Santa Maria, RS, 3 ago. 2007.

12. Universidade Federal de Santa Maria. Pró-Reitoria de Graduação. Comissão Permanente do Vestibular. Relação candidato x vaga - Ação Afirmativa. 29 nov. 2012. Disponível em:<http://www.coperves.ufsm.br/ concursos/vestibular_2012/arquivos/Vestibular_2012_Nova_Relacao_Candidato_Vaga.pdf $>$. Acesso em: 20 de jun. 2013.

13. Universidade Federal de Santa Maria. Manual do Candidato 2012. Pró-Reitoria de Graduação, Santa Maria, 2012. Disponível em:<http://www.coperves.ufsm.br/concursos/vestibular_2012/arquivos/Manual_ do_Candidato_Concurso_Vestibular_2012.pdf >. Acesso em: 20 de jan. de 2013.

14. Organização Mundial da Saúde, CIF: Classificação Internacional de Funcionalidade, Incapacidade e Saúde [Centro Colaborador da Organização Mundial da Saúde para a Família de Classificações Internacionais, org.; coordenação da tradução Cassia Maria Buchalla. São Paulo: Editora da Universidade de São Paulo EDUSP; 2003.

15. Farias N, Buchalla CMA. Classificação Internacional de Funcionalidade, Incapacidade e Saúde da Organização Mundial da Saúde: conceitos, usos e perspectivas. Revista Brasileira de Epidemiologia, São Paulo, 2005; 8 (2): 187-193.

16. Ponte AS. O cotidiano e a condição laboral de sujeitos acometidos por Lesão Encefálica Adquirida. [Dissertação de Mestrado]. Santa Maria (RS): Universidade Federal de Santa Maria. Santa Maria; 2015.

17. Ponte AS, Duarte BSL, Godoy MCM, Delboni MCC, Costa TR. Síndrome Complexa de Dor Regional do tipo I do membro superior: tratamento baseado no Estresse Muscular de Tração e Compressão Ativa. Cad. Ter. Ocup. UFSCar, São Carlos, 2015; 23(1): 3-13.

18. Pommerehn J, Delboni MCC, Fedosse E. Classificação Internacional De Funcionalidade, Incapacidade e Saúde e Afasia: Um Estudo Da Participação Social. Rev CoDas. 2015:28(2).

19. Censo 2010: escolaridade e rendimento aumentam e cai mortalidade infantil. IBGE. Rio de Janeiro, abr. 2012. Disponível em: http://www.ibge.gov.br/home/presidencia/noticias/noticia_visualiza.php?id noticia=2125\&id_pagina=1. Acesso em: 05 jul. 2013.

20. Oliver M. The politics of disablement. Basingstoke, Macmillan and St Martin's Press, 1990

21. Diniz D. O que é deficiência? São Paulo: Brasiliense, 2007.

22. Rocha TB, Miranda TG. Acesso e permanência do aluno com deficiência na instituição de ensino superior. Revista Educação Especial, Santa Maria, 2009;22(34): 197-212. 